\title{
The ability of human nuclear DNA to cause false positive low-abundance heteroplasmy calls varies across the mitochondrial genome
}

Levent Albayrak ${ }^{1,2,3}$, Kamil Khanipov1,2,3, Maria Pimenova ${ }^{1,2}$, George Golovko ${ }^{1,2}$, Mark Rojas ${ }^{1,2}$, Ioannis Pavlidis ${ }^{3}$, Sergei Chumakov ${ }^{4}$, Gerardo Aguilar ${ }^{4}$, Arturo Chávez ${ }^{4}$, William R. Widger ${ }^{5}$ and Yuriy Fofanov ${ }^{1,2^{*}}$

\begin{abstract}
Background: Low-abundance mutations in mitochondrial populations (mutations with minor allele frequency $\leq 1 \%$ ), are associated with cancer, aging, and neurodegenerative disorders. While recent progress in high-throughput sequencing technology has significantly improved the heteroplasmy identification process, the ability of this technology to detect low-abundance mutations can be affected by the presence of similar sequences originating from nuclear DNA (nDNA). To determine to what extent nDNA can cause false positive low-abundance heteroplasmy calls, we have identified mitochondrial locations of all subsequences that are common or similar (one mismatch allowed) between nDNA and mitochondrial DNA (mtDNA).

Results: Performed analysis revealed up to a 25-fold variation in the lengths of longest common and longest similar (one mismatch allowed) subsequences across the mitochondrial genome. The size of the longest subsequences shared between $\mathrm{nDNA}$ and mtDNA in several regions of the mitochondrial genome were found to be as low as 11 bases, which not only allows using these regions to design new, very specific PCR primers, but also supports the hypothesis of the non-random introduction of mtDNA into the human nuclear DNA.

Conclusion: Analysis of the mitochondrial locations of the subsequences shared between nDNA and mtDNA suggested that even very short (36 bases) single-end sequencing reads can be used to identify low-abundance variation in $20.4 \%$ of the mitochondrial genome. For longer (76 and 150 bases) reads, the proportion of the mitochondrial genome where nDNA presence will not interfere found to be 44.5 and $67.9 \%$, when low-abundance mutations at $100 \%$ of locations can be identified using 417 bases long single reads. This observation suggests that the analysis of low-abundance variations in mitochondria population can be extended to a variety of large data collections such as NCBI Sequence Read Archive, European Nucleotide Archive, The Cancer Genome Atlas, and International Cancer Genome Consortium.
\end{abstract}

Keywords: Mitochondria, Heteroplasmy, Minor allele, Low-abundance mutation, NUMT, High throughput sequencing, Rare variant

\footnotetext{
* Correspondence: yufofano@UTMB.EDU

'Department of Pharmacology and Toxicology, University of Texas Medical

Branch, 301 University Boulevard, Galveston, TX 77555-0144, USA

${ }^{2}$ Sealy Center for Structural Biology and Molecular Biophysics, University of

Texas Medical Branch, Galveston, TX, USA

Full list of author information is available at the end of the article
} 


\section{Background}

A mitochondrion has double-stranded circular DNA, which encodes 37 genes essential for normal cell functions such as cellular energy metabolism, free radical generation, and apoptosis [1-3]. Due to its high mutation rate and only rudimentary DNA repair capabilities [4], mtDNA sequences vary among individuals, organs, and tissues $[5,6]$. Furthermore, de-novo mtDNA mutations can accumulate over the lifetime of the individual and result in progressive deterioration of mitochondrial function [7-11]. Given that there are $2-10$ copies of mtDNA per mitochondrion and up to 1000 mitochondria per cell [12], mutations in mtDNA are generally heteroplasmic, with copies of both wild-type and mutant mtDNA in each cell [13]. Low-level heteroplasmy, mitochondrial DNA mutations with minor allele frequency $\leq 1 \%$, is associated with aging [14], cancer [15], and neurodegenerative disorders such as Alzheimer's [16] and Parkinson's disease [17].

Most of the techniques traditionally used to detect heteroplasmy such as Sanger capillary sequencing [18], high-performance liquid chromatography [19], SNaPshot [20], high-resolution melt profiling [21], temporal temperature gradient gel electrophoresis [22], Invader assay [23], and surveyor nuclease digestion [24] require the candidate positions to be pre-defined and do not allow determination of de-novo heteroplasmic locations. High Throughput Sequencing (HTS) technology allows detection of heteroplasmy across multiple locations in the mitochondrial genome simultaneously, making it the technology of choice in recent studies [13, 25-27].

However, the ability of this technology to detect heteroplasmy, especially low-abundance mutations, has its limitations. While some studies suggest that false positive rare variants can be artifacts of the sequencing technology [28] and mapping algorithms (software) [29-32], many publications have also focused on the interference of nuclear sequences of mitochondrial origin (NUMTs) on the detection of rare variants [33-35]. These studies generally consider variants with abundance below $2 \%$ potentially false positive and exclude them. The landmark work by $\mathrm{Li}$ et al. [28] for example, used a large number of already identified NUMTs to estimate the accuracy of low-level heteroplasmy calls and distinguish them from sequencing errors. This approach, however, relies on the reference database of NUMTs used in the analysis.

It is important to emphasize that while using only NUMTs to identify possible locations in the mitochondrial genome where nDNA can cause false positive heteroplasmy makes the computational task relatively easy, the search for NUMTs in human nuclear genomes is not yet over. Long and highly similar sequences shared between nuclear and mitochondrial DNA, also called high fidelity NUMTs are well described [36]. The search for new NUMTs focused on shorter and less similar subsequences continues [37, 38]. The results (potential new NUMTs) however, vary depending on the sequence similarity threshold, alignment length, and types of search algorithms used in the analysis [38].

To date, the use of paired end sequencing reads is believed to be the best way to avoid nDNA interference by making sure that both reads are mapped to the mitochondrial genome with appropriate distance between them. This assumption, however, does not take into consideration that at least 18 known NUMTs are longer that 5000 bases (out of which four are longer than 10,000 bases with the longest known to date is of the size of 14,904 bases) [39]. These NUMTs are able to produce reads pairs that may mistakenly be attributed as originating from mtDNA.

An alternative approach to minimize the effects of unknown (unidentified) NUMTs is including a "nuclear DNA exclusion" step into the heteroplasmy detection workflow. The basic idea of this method is to map all sequencing reads to the nDNA and completely exclude them from the analysis [7, 34, 35]. This approach is computationally expensive: sequencing reads from each experiment have to be mapped to approximately three gigabases long human nuclear genome. Additionally, the outcome of this approach will be significantly affected by the presence of short (starting from 11 bases) and very similar regions shared between mtDNA and nDNA sequences including, but not limited to known NUMTs. The longest shared (no mismatches) subsequence between nDNA and mtDNA is 279 bases (starting at position 4457 in the mitochondrial genome (NC_012920.1) and at position 629,627 in chromosome 1). Allowing just one mismatch, the same region of chromosome 1 extends to 417 bases (starting from positions 629,489 in chromosome 1 and position 4319 in mitochondria). The main disadvantage of the "nuclear DNA exclusion" approach is its exclusion of sequencing reads originating from such regions from consideration. This step artificially reduces coverage of the corresponding mtDNA regions and may result in false negative (missing heteroplasmy variants) outcomes.

To eliminate challenges of both approaches (using only known NUMTs and nuclear DNA exclusion) we propose to pre-compute the locations of all subsequences in the mitochondrial genome shared perfectly (no mismatches) and approximately (one mismatch allowed) between nDNA and mtDNA. This information allows to (a) avoid the nuclear DNA exclusion step so the reads only need to be mapped to the much smaller mitochondrial genome; (b) exclude ambiguous (mapped simultaneously to nuclear and mitochondrial DNA) reads; and (c) eliminate discrepancies 
due to the incompleteness and subjectivity in the choice of NUMTs data used in the analysis.

\section{Results and discussion Interference maps}

Exhaustive comparisons of all DNA subsequences present in the human nuclear and reference mitochondrial genomes resulted in the creation of two nuclear interference maps for the mitochondrial genome: the exact match map and the approximate match map (Fig. 1, and Additional file 1). The exact match map assigns to each position in the mitochondrial genome the length of the longest common subsequence (LCS): the longest subsequence, which includes given position in the mitochondrial genome and present simultaneously (without mismatches) in both nuclear and mitochondrial genomes. The approximate match map assigns to each position in the mitochondrial genome the length of the longest similar subsequence (LSS): the longest subsequence, which includes given position in the mitochondrial genome and present simultaneously (with up to one mismatch difference) in both nuclear and mitochondrial genomes.

As expected, due to the presence of NUMTs and other regions containing subsequences similar to the mitochondrial genome, some common/similar subsequences between mitochondrial and nuclear DNA appear to be quite long: longest LCS and LSS among all locations in the mitochondrial genome are 279 and 417 bases in length, respectively. Performed calculations (Table 1) revealed the presence of long LCS and LSS in highly studied genes such as ND2 (NADH dehydrogenase 2), COX1 (cytochrome c oxidase I), MT-COX2 (cytochrome c oxidase II), ATP6 (ATP synthase 6), as well as ATP8 (ATP synthase protein 8 ). This observation suggested that in order to avoid nDNA interference in every location in these genes, single end reads used in sequencing experiment have to be longer that 417 bases: the maximal length of LSS. In contrast, longest and average LCS and LSS in CYTB (cytochrome b), ND3 (NADH dehydrogenase 3), and ND4L (NADH-ubiquinone oxidoreductase chain $4 \mathrm{~L}$ ) genes appeared much shorter, all nDNA interference in these genes can be avoided by using single end reads as short as 100 bases. In fact, the frequency distribution of the LSS across the mitochondrial genome suggests that for approximately $20.4 \%$ using only 36 bases long single end reads will be enough to avoid the nDNA interference (44.5 and $67.9 \%$ for 76 and 150 bases long reads, respectively). This opens the opportunity to extend analysis of

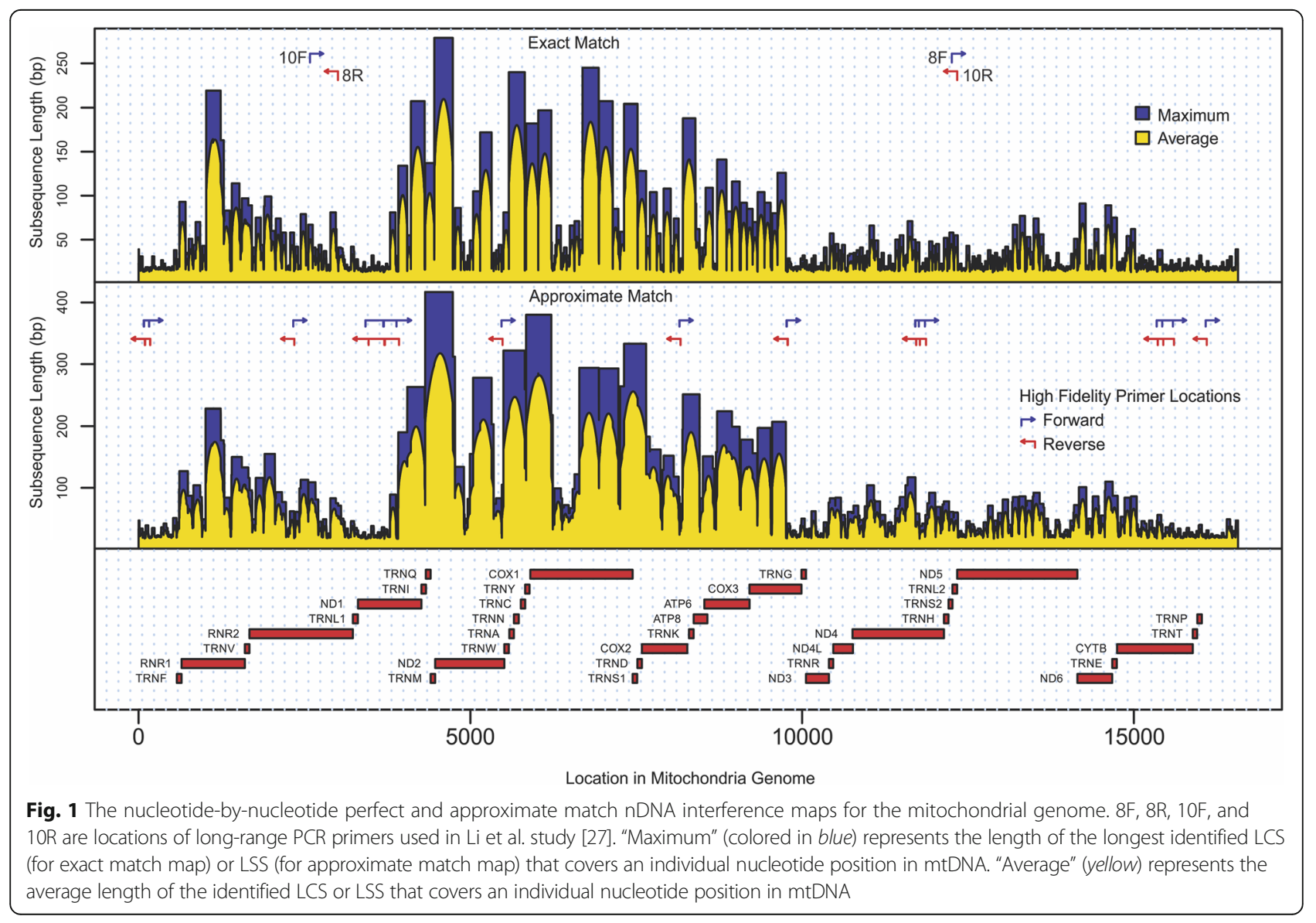


Table 1 Longest common and longest similar subsequences in mitochondrial genes

\begin{tabular}{|c|c|c|c|c|c|c|c|}
\hline Gene Name & Length & Start Position & End Position & $\begin{array}{l}\text { Maximum Length of } \\
\text { Longest Common } \\
\text { Subsequences }\end{array}$ & $\begin{array}{l}\text { Average Length of } \\
\text { Longest Common } \\
\text { Subsequences }\end{array}$ & $\begin{array}{l}\text { Maximum Length } \\
\text { of Longest Similar } \\
\text { Subsequences }\end{array}$ & $\begin{array}{l}\text { Average Length } \\
\text { of Longest Similar } \\
\text { Subsequences }\end{array}$ \\
\hline TRNF & 71 & 577 & 647 & 93 & 55.2 & 127 & 85.2 \\
\hline RNR1 & 954 & 648 & 1601 & 219 & 108.3 & 228 & 139.9 \\
\hline TRNV & 69 & 1602 & 1670 & 97 & 95.0 & 133 & 128.8 \\
\hline RNR2 & 1559 & 1671 & 3229 & 99 & 51.3 & 155 & 81.1 \\
\hline TRNL1 & 75 & 3230 & 3304 & 42 & 28.6 & 46 & 37.3 \\
\hline ND1 & 956 & 3307 & 4262 & 207 & 74.0 & 263 & 108.6 \\
\hline TRNI & 69 & 4263 & 4331 & 207 & 174.8 & 417 & 285.9 \\
\hline TRNQ & 72 & 4400 & 4329 & 137 & 137.0 & 417 & 417.0 \\
\hline TRNM & 68 & 4402 & 4469 & 279 & 162.7 & 417 & 417.0 \\
\hline ND2 & 1042 & 4470 & 5511 & 279 & 131.9 & 417 & 242.3 \\
\hline TRNW & 68 & 5512 & 5579 & 81 & 81.0 & 322 & 322.0 \\
\hline TRNA & 69 & 5655 & 5587 & 240 & 240.0 & 322 & 322.0 \\
\hline TRNN & 73 & 5729 & 5657 & 240 & 240.0 & 322 & 322.0 \\
\hline TRNC & 66 & 5826 & 5761 & 240 & 219.8 & 322 & 316.3 \\
\hline TRNY & 66 & 5891 & 5826 & 182 & 144.7 & 380 & 351.6 \\
\hline COX1 & 1542 & 5904 & 7445 & 245 & 145.7 & 380 & 255.8 \\
\hline TRNS1 & 69 & 7514 & 7446 & 204 & 204.0 & 333 & 333.0 \\
\hline TRND & 68 & 7518 & 7585 & 204 & 129.8 & 333 & 333.0 \\
\hline $\operatorname{cox} 2$ & 684 & 7586 & 8269 & 188 & 86.4 & 333 & 169.4 \\
\hline TRNK & 70 & 8295 & 8364 & 188 & 188.0 & 251 & 251.0 \\
\hline ATP8 & 207 & 8366 & 8572 & 188 & 76.2 & 251 & 177.3 \\
\hline ATP6 & 681 & 8527 & 9207 & 141 & 97.4 & 224 & 183.6 \\
\hline $\operatorname{cox} 3$ & 784 & 9207 & 9990 & 126 & 71.4 & 207 & 140.4 \\
\hline TRNG & 68 & 9991 & 10058 & 32 & 29.4 & 50 & 45.0 \\
\hline ND3 & 346 & 10059 & 10404 & 41 & 24.4 & 76 & 36.6 \\
\hline TRNR & 65 & 10405 & 10469 & 57 & 49.4 & 83 & 81.6 \\
\hline ND4L & 297 & 10470 & 10766 & 57 & 33.6 & 84 & 63.6 \\
\hline ND4 & 1378 & 10760 & 12137 & 71 & 37.1 & 117 & 70.6 \\
\hline TRNH & 69 & 12138 & 12206 & 52 & 44.9 & 78 & 78.0 \\
\hline TRNS2 & 59 & 12207 & 12265 & 58 & 50.3 & 78 & 62.3 \\
\hline TRNL2 & 71 & 12266 & 12336 & 58 & 51.8 & 61 & 59.6 \\
\hline ND5 & 1812 & 12337 & 14148 & 77 & 34.1 & 92 & 53.0 \\
\hline ND6 & 525 & 14673 & 14149 & 91 & 52.4 & 110 & 78.5 \\
\hline TRNE & 69 & 14742 & 14674 & 75 & 75.0 & 110 & 89.0 \\
\hline CYTB & 1141 & 14747 & 15887 & 62 & 27.4 & 87 & 43.3 \\
\hline TRNT & 66 & 15888 & 15953 & 26 & 20.0 & 30 & 24.0 \\
\hline TRNP & 68 & 15956 & 16023 & 32 & 26.6 & 40 & 36.1 \\
\hline
\end{tabular}

low-abundance variation in mitochondrial population to large data collections such as NCBI Sequence Read Archive [40], European Nucleotide Archive [41], The Cancer Genome Atlas [42], and International Cancer Genome Consortium [43] which were by no means designed to explore the low-level heteroplasmy in mitochondrial genome.

Surprisingly, we observed more than a 25 -fold difference in the length of both LCS and LSS across different locations in the mitochondrial genome. The shortest 
LCS was only 11 bases long and appeared in two locations in the ND5 gene. The shortest LSS was 15 bases long and appeared in two locations of the hypervariable D-loop region. As one can see in Fig. 1, the locations of the LCS and LSS do not appear to be random and express a bias to particular regions of the mitochondrial genome. For example, the region located between positions 4000 and 10,000 in the mitochondrial genome contains the vast majority of long LCS and LSS while the region between positions 10,000 and 16,000 contains a high concentration of short LCS and LSS. One possible explanation of these results can be that the introduction of mitochondrial sequences into the nuclear genome has a bias against certain locations in mtDNA (such as the 10-16 k region). Another possibility is that some mtDNA sequences have been introduced into nuclear DNA over earlier periods of evolution and have accumulated more mutations than other sequences that have been more recently transferred from mtDNA to nDNA. The length distributions of LCS and LSS that almost perfectly fit into a mixture of two and three Poisson distributions (Fig. 2a-d) also suggested the involvement of several different mechanisms behind the origin/evolution of shared mtDNA and nDNA subsequences. These observations are also in agreement with recent work by Tsuji et al. [44] reporting the underrepresentation of D-Loop sequences in nDNA. Performed analysis, however, pointed to several additional locations in the mitochondrial genome (including regions within the RNR1, TRNL1, and ND1 genes) exhibiting properties similar to D-loop sequences (i.e. being distant from nDNA) suggesting that these regions could be mutated and/or underrepresented in nDNA (Fig. 1 and Table 1).

\section{Long range PCR primers}

The noticeable presence of short subsequences unique to mtDNA contradicts the common opinion that due to the NUMTs, primers designed for mtDNA amplification will always amplify (perhaps linearly) some amount of nDNA. Although this is true for the primers originally designed by Meyer et al. for use in long-range amplification [45] and employed in the Li et al. studies [27, 28, 46] (the locations of primer pairs for 8 and $10 \mathrm{~kb}$ overlapping mtDNA regions can be found in Fig. 1), performed analysis suggests that each of these aforementioned primers is not unique to mtDNA and has many similar sequences in nDNA (Table 2, Fig. 3). Furthermore, the analysis of the frequency distribution of the length of LCS and LSS revealed that 39 and $72 \%$ of locations, respectively, in the mitochondrial genome are more specific than the locations chosen for these primers. In-silico PCR, using MFEprimer-3.0 [47] against the human genome, shows that these primers can produce 24 amplicons from the human nuclear genome (see Additional file 2 for more information).

The best locations for highly specific PCR primers would be positions where the lengths of the LSS are at their minima. Several such locations, which are highly specific for mtDNA, are shown in Fig. 1. As an example, we tested four of such location and designed new longrange PCR primers (Table 2, Fig. 3) to amplify this
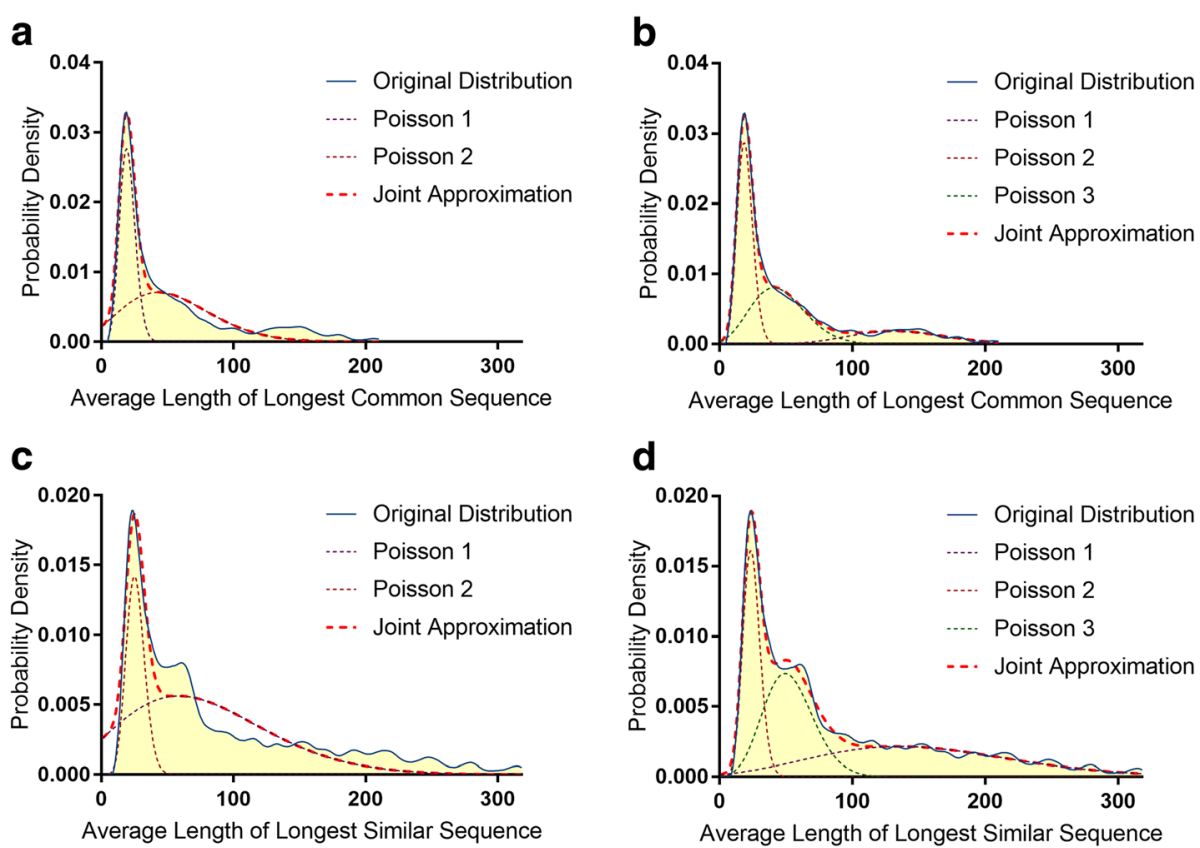

Fig. 2 Poisson distributions. Approximation of average length of LCS and LSS covering each position in the mitochondrial genome by two (a, c) and three $(\mathbf{b}, \mathbf{d})$ mixed Poisson distributions. The red dashed line represents the joint approximation of the mixed distributions 
Table 2 Characteristics of four long-range mtDNA PCR primers used by Li et al [27] and four proposed high fidelity long-range primers

\begin{tabular}{|c|c|c|c|c|c|c|}
\hline Primers set & Primer & Sequence & $\begin{array}{l}\text { Number of similar } \\
\text { sequences in } \\
\text { nDNA }\end{array}$ & $\begin{array}{l}\text { Primer length/ } \\
\text { LSS starting from } \\
\text { primers position }\end{array}$ & $\begin{array}{l}\text { Number of positions } \\
\text { in mtDNA with } \\
\text { less value of LSS }\end{array}$ & $\begin{array}{l}\text { Percent of positions } \\
\text { in mtDNA with } \\
\text { less value of LSS }\end{array}$ \\
\hline \multirow{4}{*}{$\begin{array}{l}\text { Long-range mtDNA } \\
\text { PCR primers used } \\
\text { by Li et al [25] }\end{array}$} & $\begin{array}{l}10 \mathrm{~F} \\
\text { (forward_10kb) }\end{array}$ & CCGTGCAAAGGTAGCATAATC & 8 & $21 / 84$ & 11,965 & $72.21 \%$ \\
\hline & $\begin{array}{l}10 \mathrm{R} \\
\text { (reverse_10kb) }\end{array}$ & TTACTITATTTGGAGTTGCACCA & 16 & $24 / 52$ & 9,125 & $55.07 \%$ \\
\hline & $\begin{array}{l}8 \mathrm{~F} \\
\text { (forward_8kb) }\end{array}$ & GGCTTTCTCAACTITTAAAGGATA & 10 & $24 / 34$ & 6,504 & $39.25 \%$ \\
\hline & $\begin{array}{l}8 \mathrm{R} \\
\text { (reverse_8kb) }\end{array}$ & TGTCCTGATCCAACATCGAG & 14 & $20 / 72$ & 11,089 & $66.93 \%$ \\
\hline \multirow{4}{*}{$\begin{array}{l}\text { Proposed long- } \\
\text { range mtDNA } \\
\text { PCR primers }\end{array}$} & $\begin{array}{l}\text { AF } \\
\text { (forward_10kb) }\end{array}$ & TACTACCAGACAACCTTAGCCA & 0 & $22 / 18$ & 218 & $1.32 \%$ \\
\hline & $\begin{array}{l}\text { AR } \\
\text { (reverse_10kb) }\end{array}$ & GAGTCATAAGTGGAGTCCGTAA & 0 & $22 / 18$ & 217 & $1.31 \%$ \\
\hline & $\begin{array}{l}\text { BF } \\
\text { (forward_8kb) }\end{array}$ & CACCATTTCCGACGGCATCTAC & 0 & $22 / 16$ & 1 & $0.01 \%$ \\
\hline & $\begin{array}{l}\text { BR } \\
\text { (reverse_8kb) }\end{array}$ & TGCGCCAGGTTTCAATITCTAT & 0 & $22 / 18$ & 217 & $1.31 \%$ \\
\hline
\end{tabular}

region. In-silico PCR using these primers and a human genome target shows no viable nDNA amplicons (see Additional file 2 for more information). An in-vivo validation using Illumina MiSeq at 151 cycles followed by mapping to the mitochondrial genome (NC_012920.1) and the human genome (GRCh38) shows that the proposed primers allowed a $10 \%$ reduction in the proportion of reads mapped to the nuclear genome at 95\% alignment identity (see Methods section for more details). It is important to emphasize, however, that proposed primers are only an example of how the fidelity of long-range PCR can be improved. Figure 1 shows several (out of many) possible locations for long-range PCR primers. Testing all the mitochondria-specific locations could lead to further improvement of the mtDNA enrichment protocol but it is beyond the scope of this manuscript.

It is important to emphasize the limitations of the presented interference maps. The latest human genome

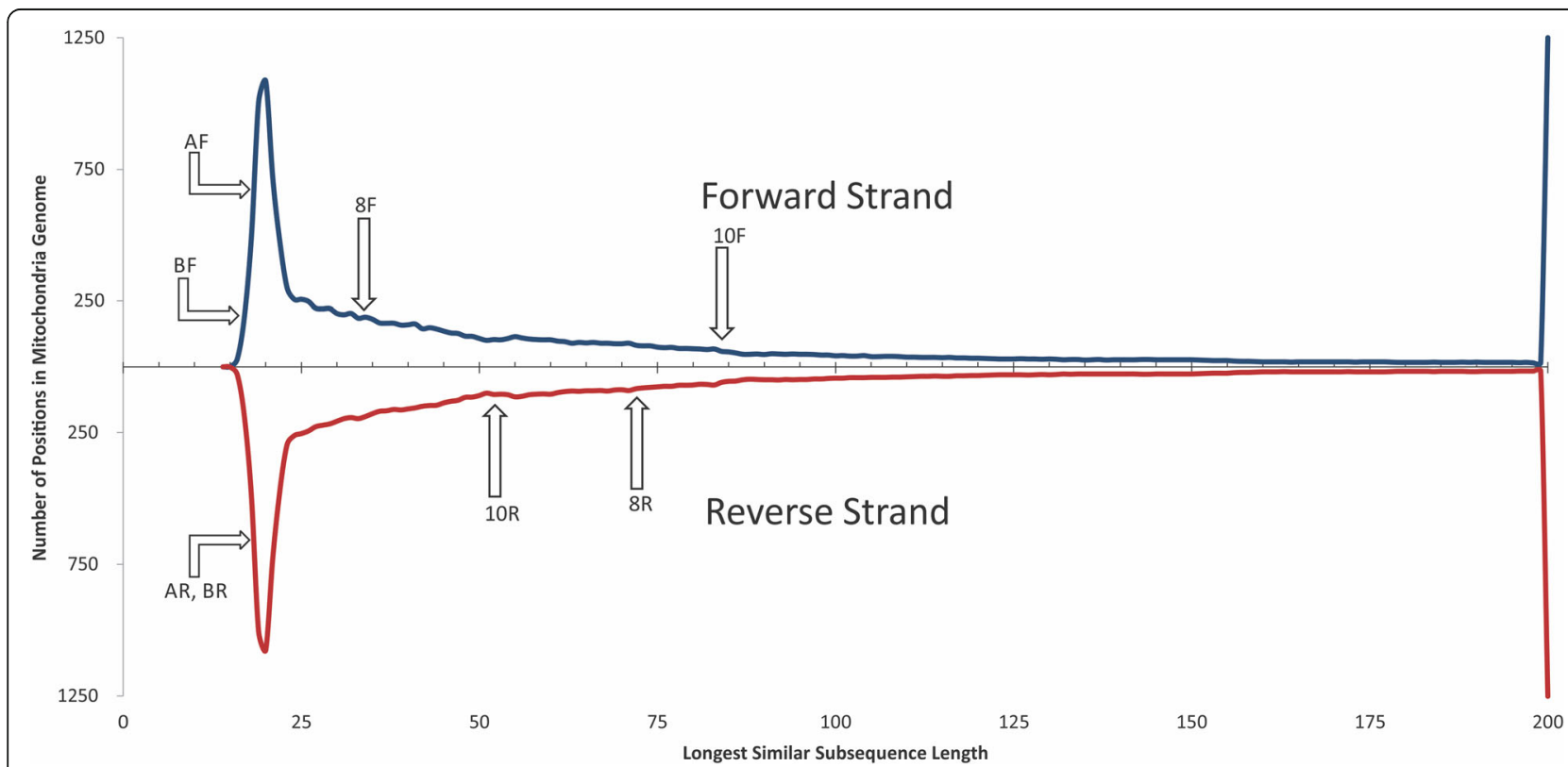

Fig. 3 The length distribution of LSS in the mitochondrial genome. 8F, 8R, 10F, and 10R are locations of forward (F) and reverse (R) primers used for long-range mtDNA amplification by Li et al [27]. AF, AR, BF, and BR are the primers designed to be located in the regions with the lowest similarity to the nuclear genome 
build (GRCh38) used in the analysis is missing some sequences from highly repetitious regions, especially centromeres. Additionally, personal genomic variations (single nucleotide polymorphisms and structural variations) and possible variations in NUMTs between individuals were not included in our computations. More robust interference maps can be built using a higher number of mismatches. For these reasons, the presented interference maps only allow the identification of the minimum required sequencing read length in mitochondrial regions of interest and establishes a confidence level in the analysis results.

\section{Conclusion}

Due to high throughput and rapidly decreasing cost, HTS offers new opportunities to identify mitochondrial heteroplasmy including low-level heteroplasmy. The presence of a significant amount of nDNA, however, can introduce bias and cause false positive low-level heteroplasmy calls. This is especially important for in-solution capturing techniques, which have become more popular for mitochondrial heteroplasmy analysis over the last few years [48-51].

To avoid interference from nDNA at each given location, the length of the LSS at that location must be longer than the length of the sequencing reads. Proposed maps allow to predict the level of possible nDNA interference for each location in the mitochondrial genome, improve the fidelity of rare variant identification, and guide the choice of the sequencing read length. Performed analysis suggests that heteroplasmy (including low-level heteroplasmy) can be identified using short (36, 76, and 150 bases long) single end read for the significant part of the mitochondrial genome, which opens an opportunity to employ the data from a variety of existing collections such as the Sequence Read Archive and The Cancer Genome Atlas into the analysis.

Identified locations of short LCS and LSS in the mitochondrial genome can also be used to improve the quality of long-range PCR primers and probes for in-solution mtDNA capture by eliminating binding to the nuclear genome. The locations and length distributions of LCS and LSS support the hypothesis of non-random mtDNA introduction into human nuclear DNA and suggest that several regions of the mitochondrial genome (in addition to the previously reported D-loop) may be underrepresented or mutated after being introduced into nDNA.

\section{Methods}

Nuclear, mitochondrial, and NUMTs data

Presented analyses were performed using the human reference genome (GRCh38). In addition to the sequences of the 24 chromosomes, this build contains 430 unlocalized scaffolds (genomic subsequences with an identified chromosome of origin but an unknown location or orientation) totaling $120,999,704$ bases in length. It is important to emphasize that the widely accepted mitochondrial reference genome (NC_012920.1) used in the presented analysis contains an unknown nucleotide "N" (position 3106) which was artificially introduced during the revision of the Cambridge reference sequence of human mitochondrial DNA [2] to maintain position numbering consistent with previous literature [2]. During mapping and analysis, this nucleotide was removed from the reference sequence and restored after mapping. The longest common and longest similar sequence lengths for this position were assigned to be equal to the corresponding values for the previous (3105) position. The NUMTs database uploaded by Simone et al. [38] in an unpublished study, "Revised RHNumtS compilation," was downloaded from the NCBI Nucleotide database [39] in June 2015. The list of accession numbers for the NUMTs is available in the Additional file 2 document.

\section{HTS data and analysis}

In order to find the LCS and LSS between human nuclear DNA and mitochondrial DNA, all human reference sequences were disassembled into overlapping subsequences of 450 bases so that each location in the human genome was associated with two (original and reverse complement) disassembled subsequences. The initial choice of the length of sequences used in the analysis (450 bases) has been made under the assumption that the maximum length of LSS will be lower than this value (which appeared to be 417). Otherwise, this value would be increased and calculations would be repeated. Humanderived subsequences were de-replicated (duplications excluded) for future calculations. The nDNA subsequences were placed in a search efficient data structure (combination of a sorted array and suffix tree) allowing to perform an exhaustive search with mismatches (insertions, deletions, and substitutions) for each subsequence and all of its prefixes. Each prefix of every 450 base-long subsequence derived from the mitochondrial genome (including complementary sequence) was searched against this data structure with perfect matching to identify the LCS and with one permitted mismatch to identify the LSS starting from each position. Software specifically developed for this purpose was used in the computations performed on the Lonestar high-performance computing cluster at the Texas Advanced Computing Center (The University of Texas at Austin).

The interference maps were created by identifying each of the longest common and longest similar subsequences that included the given position and calculated the maximum and average length of such subsequences (Fig. 1, and Additional file 1). 
Table 3 Error and parameter values identified for average length of LCS and LSS distributions using two and three Poisson mixtures

\begin{tabular}{|c|c|c|c|c|}
\hline \multirow[t]{2}{*}{$\bar{x}$} & \multicolumn{2}{|l|}{ LCS } & \multicolumn{2}{|l|}{ LSS } \\
\hline & $\begin{array}{l}\text { Mixture of } 2 \text { Poisson Distributions } \\
(k=2)\end{array}$ & $\begin{array}{l}\text { Mixture of } 3 \text { Poisson Distributions } \\
(k=3)\end{array}$ & $\begin{array}{l}\text { Mixture of } 2 \text { Poisson Distributions } \\
(k=2)\end{array}$ & $\begin{array}{l}\text { Mixture of } 3 \text { Poisson Distributions } \\
(k=3)\end{array}$ \\
\hline$a_{1}$ & 0.4071 & 0.1709 & 0.7556 & 0.3993 \\
\hline$a_{2}$ & 0.5929 & 0.4195 & 0.2444 & 0.2574 \\
\hline$a_{3}$ & & 0.4097 & & 0.3433 \\
\hline$\beta_{1}$ & 0.5729 & 0.1030 & 0.0263 & 0.0277 \\
\hline$\beta_{2}$ & 0.0472 & 0.5691 & 0.5456 & 0.5980 \\
\hline$\beta_{3}$ & & 0.1090 & & 0.1511 \\
\hline$\lambda_{1}$ & 11.3771 & 14.0520 & 2.0610 & 4.2692 \\
\hline$\lambda_{2}$ & 2.5450 & 11.0717 & 14.1339 & 14.5746 \\
\hline$\lambda_{3}$ & & 4.96376 & & 7.9806 \\
\hline error & 2.8675E-04 & 3.3724E-05 & 4.9989E-04 & 3.6505E-05 \\
\hline
\end{tabular}

\section{Poisson fitting}

A Gaussian convolution kernel filter $(\mu=21$ and $\sigma=3)$ was used to reduce the effects of noise in the probability density functions of average LCS and LSS lengths (Fig. 2). The statistical models of the distribution were defined as mixtures of two and three linearly transformed Poisson distributions where the probability density of LSS and LCS of the length $(l)$ was defined as:

$$
G(l, \alpha, \beta, \lambda, k)=\sum_{i=1}^{k} \alpha_{i} \beta_{i} P\left(\lambda_{i}, \beta_{i} l\right)
$$

where

$$
\begin{aligned}
& \alpha_{1}, . ., \alpha_{2}=\text { proportions of Poisson distributions in the } \\
& \text { mixture } \sum_{i=1}^{k} \alpha_{i}=1 ; \\
& \beta_{1}, . ., \beta_{2}=\text { transformation coefficients of Poisson } \\
& \text { distribution; } \\
& \lambda_{1}, . ., \lambda_{2}=\text { parameters of Poisson distributions; } \\
& k=\text { number of Poisson distributions in the mixture. }
\end{aligned}
$$

The fitness function was defined as the sum of squares of deviations of the model from the observed probability density and was optimized using all $3 \mathrm{k}-1$ parameters of the mixture of transformed Poisson distributions $(N=n u m-$ ber of observations, $F\left(l_{i}\right)=$ probability density at length ' $i$ '):

$$
\min _{\alpha_{1}, \ldots, \alpha_{k}} \min _{\beta_{1}, . ., \beta_{k}} \min _{\lambda_{1}, . ., \lambda_{k}} \sum_{i=1}^{N}\left(F\left(l_{i}\right)-G\left(l_{i}, \alpha, \beta, \lambda, k\right)\right)^{2}
$$

The error and parameter values for when the fitness function reaches its minimum can be found in Table 3 .

\section{PCR primer design and validation}

Two pairs of primers (pair A and pair B) amplifying overlapping 10 and $8 \mathrm{~kb}$ regions, respectively, of the mitochondrial genome, were selected from regions with the lowest similarity to the nuclear genome using the proposed interference maps. Primer validation was performed using in-silico PCR using MFEprimer-3.0 [46]. The same software has been used to evaluate the primers employed by $L i$ et al. [25]. Both specific and non-specific amplification was predicted for all primers using the human reference genome (GRCh38) (see Additional file 2 for more information). An in-vivo validation of the proposed primers was performed using human DNA from a de-identified archived specimen. DNA was isolated using DNeasy Blood and Tissue kit (Qiagen, CA, USA). The isolated DNA was amplified using the LongAmp Taq PCR Kit (New England Bio Labs, MA, USA) under three conditions, varying the annealing temperature. More details including the PCR amplification conditions can be found in the Additional file 2 .

Sequencing has been performed using Illumina MiSeq instrument at 151 cycles. The read filtration step included trimming reads containing: (a) nucleotides below the quality threshold of 0.05 (using modified Richard Mott algorithm); (b) two or more unknown nucleotides; and (c) Nextera tagmentation library adapters. Reads from each dataset were mapped to the mitochondrial genome (NC_012920.1) and the human genome (GRCh38, chromosomes only) using the CLC Genomics Workbench 9.0.1 "Map Reads to Contigs" analysis tool. The proposed primers showed approximately a $10 \%$ reduction in mapping to the human genome at $95 \%$ alignment identity (see Additional file 2 for more information).

\section{Additional files}

Additional file 1: Exact and approximate match nuclear DNA interference maps for the mitochondrial genome. (XLSX $1715 \mathrm{~kb}$ )

Additional file 2: Supplementary Materials Document. (DOCX 454 kb) 


\section{Abbreviations}

HTS: High throughput sequencing; LCS: Longest common subsequence; LSS: Longest similar subsequence; mtDNA: Mitochondrial DNA; nDNA: Nuclear DNA; NUMT: Nuclear sequence of mitochondrial origin

\section{Acknowledgements}

Not applicable.

\section{Funding}

YF, GG, MR, KK, MP, and LA work was partially supported by the US Defense Threat Reduction Agency, the Sealy Center for Structural Biology \& Molecular Biophysics and the Institute for Human Infections and Immunity at the University of Texas Medical Branch and Texas Advanced Computing Center. SC work was supported in part by the CONACYT (Mexico) project No. 84358.

\section{Availability of data and materials}

The sequencing datasets generated during the current study are available from the corresponding author on reasonable request.

\section{Authors' contributions}

YF, WW, LA, IP, and SC designed the experiments and interpreted the results of all analyses. LA, KK, GG, MR, MP, GA, and AC developed the analysis tools and analyzed the sequence data. LA, YF, WW, KK, and MP wrote the manuscript and GG, MR, and IP edited the manuscript. All authors read and approved the final manuscript.

\section{Competing interests}

The authors declare that they have no competing interests.

\section{Consent for publication}

Not applicable.

\section{Ethics approval and consent to participate}

The de-identified human sample used in this study has been acquired from an existing collection.

\section{Author details}

'Department of Pharmacology and Toxicology, University of Texas Medical Branch, 301 University Boulevard, Galveston, TX 77555-0144, USA. ${ }^{2}$ Sealy Center for Structural Biology and Molecular Biophysics, University of Texas Medical Branch, Galveston, TX, USA. D. Department of Computer Science, University of Houston, Houston, TX, USA. ${ }^{4}$ Department of Physics, University of Guadalajara, Guadalajara, Jalisco, Mexico. ${ }^{5}$ Department of Biology and Biochemistry, University of Houston, Houston, TX, USA.

\section{Received: 4 January 2016 Accepted: 5 December 2016}

\section{Published online: 12 December 2016}

\section{References}

1. Modica-Napolitano JS, Kulawiec M, Singh KK. Mitochondria and human cancer. Curr Mol Med. 2007;7(1):121-31.

2. Andrews RM, Kubacka I, Chinnery PF, Lightowlers RN, Turnbull DM, Howell N. Reanalysis and revision of the Cambridge reference sequence for human mitochondrial DNA. Nat Genet. 1999;23(2):147.

3. Verma M, Kumar D. Application of mitochondrial genome information in cancer epidemiology. Clin Chim Acta. 2007;383(1-2):41-50.

4. Bogenhagen DF. Repair of mtDNA in vertebrates. Am J Hum Genet. 1999. 64(5):1276-81

5. Macmillan C, Lach B, Shoubridge EA. Variable distribution of mutant mitochondrial DNAs (tRNA(Leu[3243])) in tissues of symptomatic relatives with MELAS: the role of mitotic segregation. Neurology. 1993;43(8):1586-90.

6. Chinnery PF. Mitochondrial disorders overview. 2014

7. Goto H, Dickins B, Afgan E, Paul IM, Taylor J, Makova KD, Nekrutenko A. Dynamics of mitochondrial heteroplasmy in three families investigated via a repeatable re-sequencing study. Genome Biol. 2011;12(6):R59

8. Lott MT, Leipzig JN, Derbeneva O, Xie HM, Chalkia D, Sarmady M, Procaccio V, Wallace DC. mtDNA variation and analysis using MITOMAP and MITOMASTER. Curr Protoc Bioinformatics. 2013;1(123):1-23. 21-21 2326.

9. Ramos A, Santos C, Mateiu L, Gonzalez Mdel M, Alvarez L, Azevedo L, Amorim A, Aluja MP. Frequency and pattern of heteroplasmy in the complete human mitochondrial genome. PLOS ONE. 2013;8(10):e74636.
10. Wallace DC. Mitochondrial DNA mutations in disease and aging. Environ Mol Mutagen. 2010;51(5):440-50

11. Wallace DC. A mitochondrial paradigm of metabolic and degenerative diseases, aging, and cancer: a dawn for evolutionary medicine. Annu Rev Genet. 2005;39:359-407.

12. Robin ED. Wong R. Mitochondrial DNA molecules and virtual number of mitochondria per cell in mammalian cells. J Cell Physiol. 1988;136(3):507-13.

13. He Y, Wu J, Dressman DC, lacobuzio-Donahue C, Markowitz SD, Velculescu VE, Diaz Jr LA, Kinzler KW, Vogelstein B, Papadopoulos N. Heteroplasmic mitochondrial DNA mutations in normal and tumour cells. Nature. 2010; 464(7288):610-4.

14. Smigrodzki RM, Khan SM. Mitochondrial microheteroplasmy and a theory of aging and age-related disease. Rejuvenation Res. 2005;8(3):172-98.

15. Yu J, Sadakari Y, Shindo K, Suenaga M, Brant A, Almario JA, Borges M, Barkley T, Fesharakizadeh S, Ford M et al. Digital next-generation sequencing identifies low-abundance mutations in pancreatic juice samples collected from the duodenum of patients with pancreatic cancer and intraductal papillary mucinous neoplasms. Gut. 2016. doi:10.1136/gutjnl2015-311166.

16. Lin MT, Simon DK, Ahn CH, Kim LM, Beal MF. High aggregate burden of somatic mtDNA point mutations in aging and Alzheimer's disease brain. Hum Mol Genet. 2002;11(2):133-45.

17. Parker Jr WD, Parks JK. Mitochondrial ND5 mutations in idiopathic Parkinson's disease. Biochem Biophys Res Commun. 2005;326(3):667-9.

18. Irwin JA, Saunier JL, Niederstatter H, Strouss KM, Sturk KA, Diegoli TM, Brandstatter A, Parson W, Parsons TJ. Investigation of heteroplasmy in the human mitochondrial DNA control region: a synthesis of observations from more than 5000 global population samples. J Mol Evol. 2009;68(5):516-27.

19. Meierhofer D, Mayr JA, Ebner S, Sperl W, Kofler B. Rapid screening of the entire mitochondrial DNA for low-level heteroplasmic mutations. Mitochondrion. 2005;5(4):282-96

20. Cassandrini D, Calevo MG, Tessa A, Manfredi G, Fattori F, Meschini MC Carrozzo R, Tonoli E, Pedemonte M, Minetti C, et al. A new method for analysis of mitochondrial DNA point mutations and assess levels of heteroplasmy. Biochem Biophys Res Commun. 2006;342(2):387-93.

21. Dobrowolski SF, Hendrickx AT, van den Bosch BJ, Smeets HJ, Gray J, Miller T, Sears $M$. Identifying sequence variants in the human mitochondrial genome using high-resolution melt (HRM) profiling. Hum Mutat. 2009;30(6):891-8.

22. Wong LJ, Chen TJ, Tan DJ. Detection of mitochondrial DNA mutations using temporal temperature gradient gel electrophoresis. Electrophoresis. 2004; 25(15):2602-10.

23. Mashima Y, Nagano M, Funayama T, Zhang Q, Egashira T, Kudho J, Shimizu $\mathrm{N}$, Oguchi Y. Rapid quantification of the heteroplasmy of mutant mitochondrial DNAs in Leber's hereditary optic neuropathy using the Invader technology. Clin Biochem. 2004:37(4):268-76.

24. Bannwarth S, Procaccio V, Paquis-Flucklinger V. Surveyor Nuclease: a new strategy for a rapid identification of heteroplasmic mitochondrial DNA mutations in patients with respiratory chain defects. Hum Mutat. 2005;25(6): 575-82.

25. Bai R, Higgs J, Suchy S, Arjona D, Smaoui N, Richard G, Compton J, Bale S, Parikh S, Tarnopolsky M, et al. Whole mitochondrial genome amplification and next generation sequencing for the diagnosis of mitochondrial disorders: yield of 613 cases. Mitochondrion. 2013:13(6):935-6.

26. Huang T. Next generation sequencing to characterize mitochondrial genomic DNA heteroplasmy. Curr Protoc Hum Genet. 2011:Chapter 19:Unit19.18.

27. Li M, Schonberg A, Schaefer M, Schroeder R, Nasidze I, Stoneking M. Detecting heteroplasmy from high-throughput sequencing of complete human mitochondrial DNA genomes. Am J Hum Genet. 2010;87(2):237-49.

28. Li M, Schroeder R, Ko A, Stoneking M. Fidelity of capture-enrichment for mtDNA genome sequencing: influence of NUMTs. Nucleic Acids Res. 2012; 40(18):e137.

29. Giannoulatou E, Park SH, Humphreys DT, Ho JW. Verification and validation of bioinformatics software without a gold standard: a case study of BWA and Bowtie. BMC Bioinform. 2014:15 Suppl 16:S15.

30. Hatem A, Bozdag D, Toland AE, Catalyurek UV. Benchmarking short sequence mapping tools. BMC Bioinform. 2013;14:184.

31. Li H, Homer N. A survey of sequence alignment algorithms for nextgeneration sequencing. Brief Bioinform. 2010;11(5):473-83.

32. Ruffalo M, LaFramboise T, Koyuturk M. Comparative analysis of algorithms for next-generation sequencing read alignment. Bioinformatics. 2011;27(20): 2790-6. 
33. Ye F, Samuels DC, Clark T, Guo Y. High-throughput sequencing in mitochondrial DNA research. Mitochondrion. 2014;17:157-63.

34. Guo Y, Li J, Li Cl, Shyr Y, Samuels DC. MitoSeek: extracting mitochondria information and performing high-throughput mitochondria sequencing analysis. Bioinformatics. 2013;29(9):1210-1.

35. Samuels DC, Han L, Li J, Quanghu S, Clark TA, Shyr Y, Guo Y. Finding the lost treasures in exome sequencing data. Trends Genet. 2013;29(10):593-9.

36. Lascaro D, Castellana S, Gasparre G, Romeo G, Saccone C, Attimonelli M. The RHNumtS compilation: features and bioinformatics approaches to locate and quantify Human NumtS. BMC Genomics. 2008;9:267.

37. Ramos A, Barbena E, Mateiu L, del Mar GM, Mairal Q, Lima M, Montiel R, Aluja MP, Santos C. Nuclear insertions of mitochondrial origin: database updating and usefulness in cancer studies. Mitochondrion. 2011;11(6):946-53.

38. Simone D, Calabrese FM, Lang M, Gasparre G, Attimonelli M. The reference human nuclear mitochondrial sequences compilation validated and implemented on the UCSC genome browser. BMC Genomics. 2011;12:517.

39. Calabrese FM, Simone D, Attimonelli M. Primates and mouse NumtS in the UCSC Genome Browser. BMC Bioinform. 2012;13 Suppl 4:S15.

40. Leinonen R, Sugawara H, Shumway M. The sequence read archive. Nucleic Acids Res. 2011;39:D19-21.

41. Leinonen R, Akhtar R, Birney E, Bower L, Cerdeno-Tárraga A, Cheng Y, Cleland I, Faruque N, Goodgame N, Gibson R, et al. The European nucleotide archive. Nucleic Acids Res. 2011;39:D28-31.

42. Tomczak K, Czerwińska P, Wiznerowicz M. The Cancer Genome Atlas (TCGA): an immeasurable source of knowledge. Contemp Oncol (Pozn). 2015;19: A68-77.

43. Zhang J, Baran J, Cros A, Guberman JM, Haider S, Hsu J, Liang Y, Rivkin E, Wang J, Whitty B, et al. International Cancer Genome Consortium Data Portal-a one-stop shop for cancer genomics data. Database (Oxford). 2011; 2011:bar026.

44. Tsuji J, Frith MC, Tomii K, Horton P. Mammalian NUMT insertion is nonrandom. Nucleic Acids Res. 2012;40(18):9073-88.

45. Meyer M, Stenzel U, Myles S, Prufer K, Hofreiter M. Targeted highthroughput sequencing of tagged nucleic acid samples. Nucleic Acids Res. 2007;35(15):e97.

46. Schonberg A, Theunert C, Li M, Stoneking M, Nasidze I. High-throughput sequencing of complete human mtDNA genomes from the Caucasus and West Asia: high diversity and demographic inferences. Eur J Hum Genet. 2011;19(9):988-94.

47. Qu W, Zhang C. Selecting specific PCR primers with MFEprimer. Methods Mol Biol. 2015;1275:201-13.

48. Fortes GG, Paijmans JL. Analysis of whole mitogenomes from ancient samples. Methods Mol Biol. 2015;1347:179-95.

49. Lippold S, Xu H, Ko A, Li M, Renaud G, Butthof A, Schroder R, Stoneking M. Human paternal and maternal demographic histories: insights from highresolution Y chromosome and mtDNA sequences. Investig Genet. 2014:5:13.

50. de Sousa F, Bertrand YJ, Nylinder S, Oxelman B, Eriksson JS, Pfeil BE. Phylogenetic properties of 50 nuclear loci in Medicago (Leguminosae) generated using multiplexed sequence capture and next-generation sequencing. PLoS ONE. 2014;9(10):e109704.

51. King TE, Fortes GG, Balaresque $P$, Thomas MG, Balding D, Maisano Delser $P$, Neumann R, Parson W, Knapp M, Walsh S, et al. Identification of the remains of King Richard III. Nat Commun. 2014;5:5631.

\section{Submit your next manuscript to BioMed Central and we will help you at every step:}

- We accept pre-submission inquiries

- Our selector tool helps you to find the most relevant journal

- We provide round the clock customer support

- Convenient online submission

- Thorough peer review

- Inclusion in PubMed and all major indexing services

- Maximum visibility for your research

Submit your manuscript at www.biomedcentral.com/submit
Biomed Central 